\title{
Dark Brown Hyperkeratotic Nodule on the Back
}

Amanda Kraus, MS; Ashley Elsensohn, MD, MPH; Nil Celebi Cherukuri, MD, PhD; William Tyler, MD

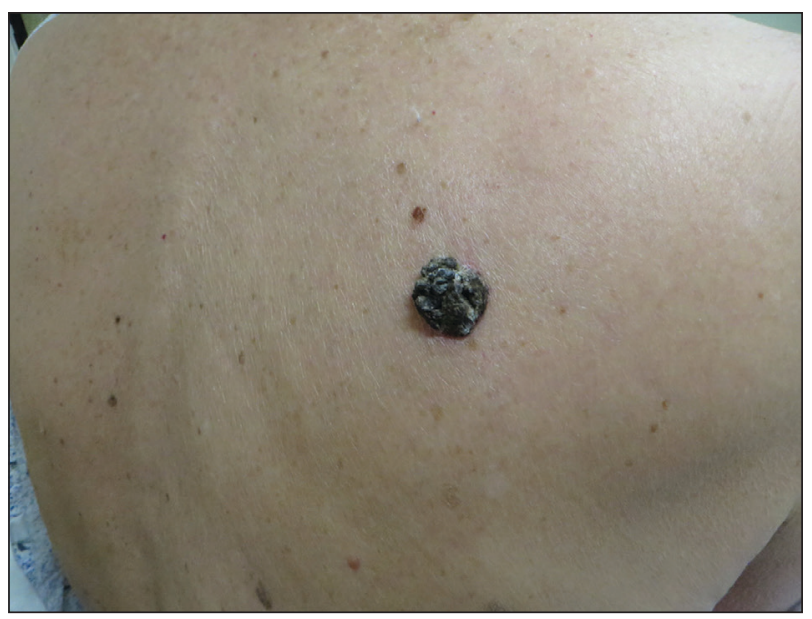

A 71-year-old woman presented with a persistent asymptomatic lesion on the right upper back that had recently increased in size and changed in color, shape, and texture. The lesion had been present for many years. Physical examination revealed a $1.5-\mathrm{cm}$, dark brown, hyperkeratotic nodule with no identifiable pigment network on dermatoscopy. The patient had no personal history of melanoma but did have a history of stage I non-small cell lung cancer. A review of systems was noncontributory. A shave biopsy of the lesion was performed.

\section{WHAT'S YOUR DIAGNOSIS?}
a. malignant hidroacanthoma simplex/
pigmented porocarcinoma
b. pigmented cutaneous metastasis
c. pigmented squamous cell carcinoma
d. seborrheic keratosis
e. seborrheic keratosis-like melanoma

Ms. Kraus is from Georgetown University School of Medicine, Washington, DC. Drs. Elsensohn and Tyler are from the Dermatopathology Section, Geisinger Medical Center, Danville, Pennsylvania. Dr. Cherukuri is from the Dermatology Section, Geisinger Medical Center, Wilkes-Barre, Pennsylvania. The authors report no conflict of interest.

Correspondence: Ashley Elsensohn, MD, MPH, Geisinger Medical Center, Laboratory Medicine, 100 N Academy Ave, MC 19-20, Danville, PA 17822 (ashley.elsensohn@gmail.com).

doi:10.12788/cutis.0237 


\section{THE DIAGNOSIS: Seborrheic Keratosis-like Melanoma}

S eborrheic keratosis (SK) is a benign neoplasm commonly encountered on the skin and frequently diagnosed by clinical examination alone. Seborrheic keratosis-like melanomas are melanomas that clinically or dermatoscopically resemble SKs and thus can be challenging to accurately diagnose. Melanomas can have a hyperkeratotic or verrucous appearance ${ }^{1-3}$ and can even exhibit dermatoscopic and microscopic features that are found in SKs such as comedolike openings and milialike cysts as well as acanthosis and pseudohorn cysts, respectively. ${ }^{2}$

In our patient, histopathology revealed SK-like architecture with hyperorthokeratosis, papillomatosis, pseudohorn cyst formation, and basaloid acanthosis (Figure).
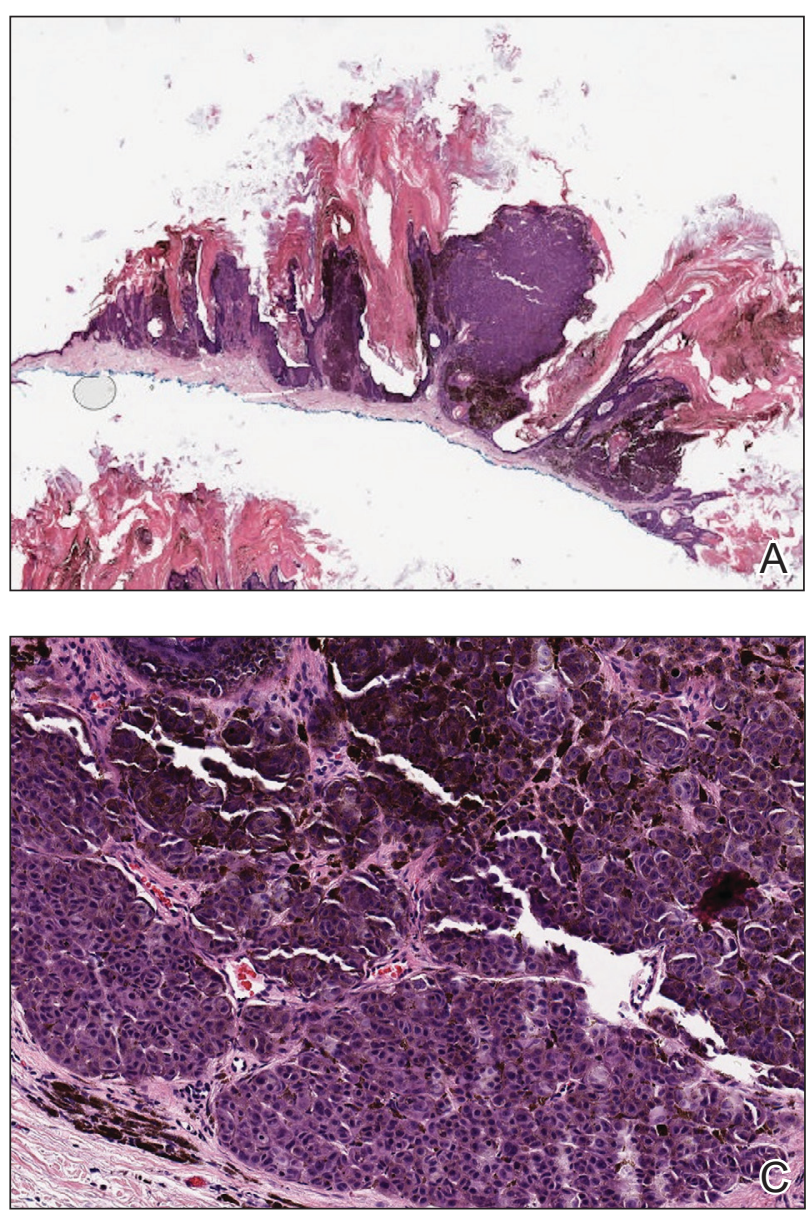

However, within the lesion was an asymmetric proliferation of nested atypical melanocytes with melanin pigment production. The atypical melanocytes filled and expanded papillomatous projections without notable pagetoid growth and extended into the dermis. There was a background congenital nevus component. These findings were diagnostic of invasive malignant melanoma, extending to a Breslow depth of $5.5 \mathrm{~mm}$. A follow-up sentinel lymph node biopsy was negative for metastatic melanoma. The clinical and histologic findings did not show melanoma in the surrounding skin to suggest colonization of an SK by an adjacent melanoma. The clinical history of a long-standing lesion in conjunction with a congenital nevus component on histology favored
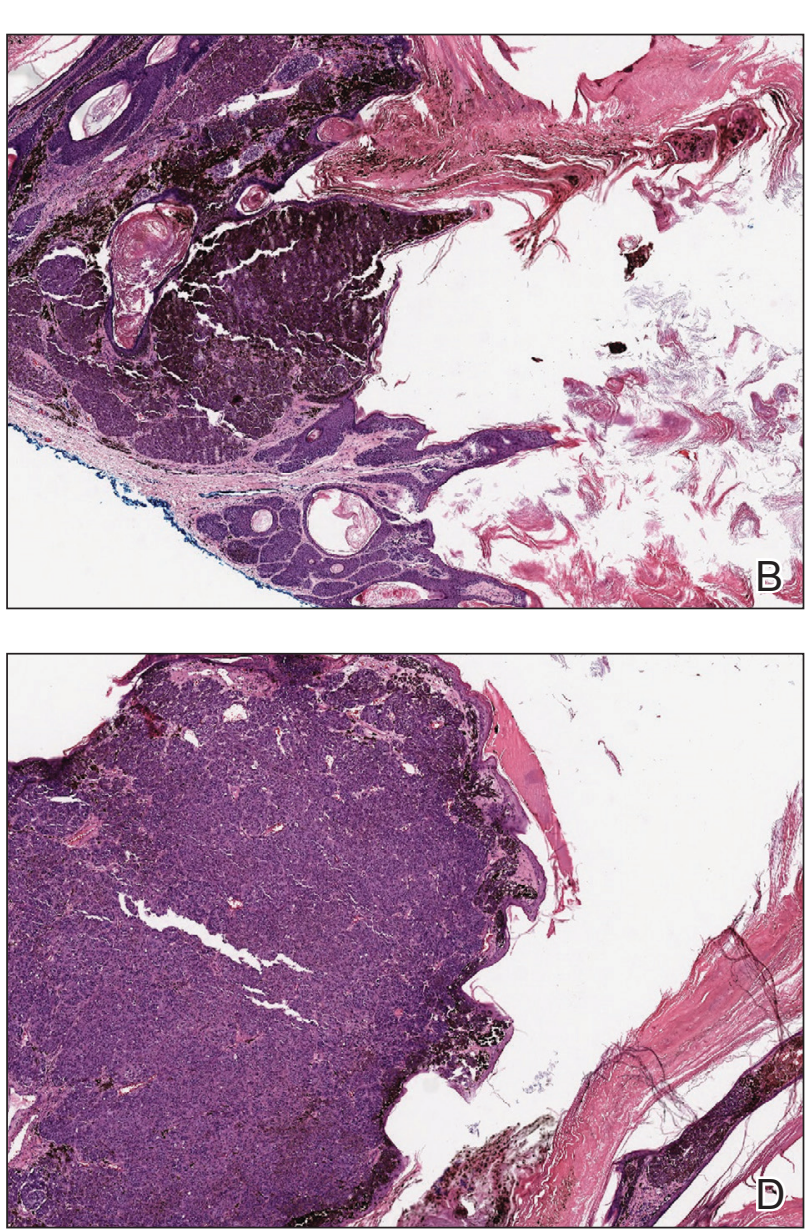

A and B, Histopathology revealed a seborrheic keratosis-like silhouette with hyperorthokeratosis, papillomatosis, pseudohorn cyst formation, and basaloid acanthosis with a concomitant proliferation of atypical melanocytic nests with abundant melanin pigment (H\&E, original magnifications $\times 10$ and $\times 20$ ). C, Melanocytic nests consisting of pleomorphic, round to epithelioid cells demonstrating marked atypia invading into the dermis, consistent with invasive malignant melanoma (H\&E, original magnification $\times 100)$. D, Atypical melanocytes filling and expanding a papillary stalk with minimal pagetoid spread $(\mathrm{H \& E}$, original magnification $\times 20)$. 
a diagnosis of melanoma arising in association with a congenital nevus with an SK-like architecture rather than arising in a preexisting SK or de novo melanoma.

Because our patient did not have multiple widespread SKs and reported rapid growth in the lesion in the last 6 months, there was concern for a malignant neoplasm. However, in patients with numerous SKs or areas of chronically sun-damaged skin, it can be difficult to identify suspicious lesions. It is important for clinicians to remain aware of SK-like melanomas and have a lower threshold for biopsy of any changing or symptomatic lesion that clinically resembles an SK. In our case, the history of change and the markedly different clinical appearance of the lesion in comparison to our patient's SKs prompted the biopsy. Criteria have been proposed to help differentiate these entities under dermoscopy, with melanoma showing the presence of the blue-black sign, pigment network, pseudopods or streaks, and/or the blue-white veil. ${ }^{4}$

Cutaneous metastases classically present as dermal nodules, plaques, or ulcers. ${ }^{5,6} \mathrm{~A}$ rare pigmented case of metastatic breast adenocarcinoma clinically mimicking melanoma has been reported. ${ }^{7}$ There is limited literature on the dermoscopic features of cutaneous metastases, but it appears that polymorphic vascular patterns are most common. ${ }^{5,8}$ The possibility of a metastatic melanoma involving an SK is a theoretical consideration, but there was no prior history of melanoma in our patient, and the histologic findings were consistent with primary melanoma. There was no histologic evidence of pigmented metastatic breast carcinoma or metastatic lung carcinoma.

Pigmented malignant hidroacanthoma simplex and pigmented porocarcinomas are rare malignant sweat gland tumors. ${ }^{9-11}$ Their benign counterparts are the more commonly encountered hidroacanthoma simplex (intraepidermal poroma) and poroma. Pigmented malignant hidroacanthoma simplex has been reported to clinically mimic an irritated SK. ${ }^{10}$ The histopathology of our case did not have features of malignant hidroacanthoma simplex or porocarcinoma. Pigmented squamous cell carcinoma is an uncommon variant of squamous cell carcinoma, and histopathology would reveal proliferation of atypical keratinocytes. ${ }^{12}$

\section{REFERENCES}

1. Saggini A, Cota C, Lora V, et al. Uncommon histopathological variants of malignant melanoma. part 2. Am J Dermatopathol. 2019;41:321-342.

2. Klebanov N, Gunasekera N, Lin WM, et al. The clinical spectrum of cutaneous melanoma morphology. J Am Acad Dermatol. 2019; 80:178-188.

3. Tran PT, Truong AK, Munday W, et al. Verrucous melanoma masquerading as a seborrheic keratosis. Dermatol Online J. 2019;25: 13030/qt1m07k7fm.

4. Carrera C, Segura S, Aguilera P. Dermoscopic clues for diagnosing melanomas that resemble seborrheic keratosis. JAMA Dermatol. 2017;153:544-551.

5. Strickley JD, Jenson AB, Jung JY. Cutaneous metastasis. Hematol Oncol Clin North Am. 2019;33:173-197.

6. Chernoff KA, Marghoob AA, Lacouture ME. Dermoscopic findings in cutaneous metastases. JAMA Dermatol. 2014;150:429-433.

7. Marti N, Molina I, Monteagudo C, et al. Cutaneous metastasis of breast carcinoma mimicking malignant melanoma in scalp. Dermatol Online J. 2008:14:12

8. Kelati A, Gallouj S. Dermoscopy of skin metastases from breast cancer: two case reports. J Med Case Rep. 2018;12:273.

9. Ishida M, Hotta M, Kushima R, et al. A case of porocarcinoma arising in pigmented hidroacanthoma simplex with multiple lymph node, liver and bone metastases. J Cutan Pathol. 2011;38:227-231.

10. Lee JY, Lin MH. Pigmented malignant hidroacanthoma simplex mimicking irritated seborrheic keratosis. J Cutan Pathol. 2006;33:705-708.

11. Ueo T, Kashima K, Daa T, et al. Porocarcinoma arising in pigmented hidroacanthoma simplex. Am J Dermatopathol. 2005;27:500-503.

12. Motta de Morais P, Schettini A, Rocha J, et al. Pigmented squamous cell carcinoma: case report and importance of differential diagnosis. An Bras Dermatol. 2018;93:96-98. 